\title{
Re-examining Voter Turnout in Large Elections*
}

\author{
Curtis R. Taylor ${ }^{\dagger}$ and Huseyin Yildirim ${ }^{\ddagger}$
}

May 2005

\begin{abstract}
A well-known shortcoming of rational voter models is that the equilibrium probability that an individual votes converges to zero as the population of citizens tends to infinity. We show that this does not - as is often suggested - imply that equilibrium voter turnout is insignificant in the limit. We characterize limiting equilibrium turnout and show that it may actually be arbitrarily large. Indeed, expected equilibrium turnout is shown to be closely approximated by $1 /\left(2 \pi \underline{c}^{2}\right)$, where $\underline{c}$ is the lowest possible realization of an individual's voting cost.
\end{abstract}

\footnotetext{
${ }^{*}$ We thank John Aldrich and Mike Munger for valuable discussions. Taylor's research was supported by NSF grant SES-0417737.

${ }^{\dagger}$ Department of Economics, Duke University, Box 90097, Durham, NC 27708-0097, email: crtaylor@econ.duke.edu

${ }^{\ddagger}$ Department of Economics, Duke University, Box 90097, Durham, NC 27708-0097, email: yildirh@econ.duke.edu
} 


\section{Introduction}

At the heart of any democracy lies the act of voting. Yet, it is paradoxically one of the least understood concepts in political economy. Given the negligible impact of an individual vote in a large electorate, it is puzzling why rational voters sacrifice their time and energy to go to the polls. Any theoretical discussion on this issue begins with the calculus of voting model (Downs 1957, Rikers and Ordeshook 1968). In this model, the expected net utility of a citizen from voting is given by

$$
\Delta U=p \Delta V+D-C,
$$

where $p$ denotes the probability of casting a decisive vote or being pivotal, $\Delta V$ is the net value from being on the winning side, $D$ is the direct benefit from the act of voting, and $C$ is the cost of voting. A citizen votes if this net utility is positive, or, equivalently, if the expected benefit, $p \Delta V$, exceeds the net cost defined as $c \equiv C-D$.

Comparing these two terms, however, is not trivial owing to the fact that the probability of being pivotal depends on voter turnout which is endogenous . In particular, this probability would be one if no one voted and close to zero if everyone in a large electorate votes. Given that most elections involve large electorates, a rational voter with a positive net cost of voting, i.e., $c>0$, will abstain. However, if this were true for every citizen, then no voting would take place, and each potential vote would in turn become pivotal with certainty. An early resolution to this "paradox of (not) voting" was proposed by Downs (1957) and subsequently refined by Rikers and Ordeshook (1968): citizens in large electorates vote despite $p \approx 0$ because they receive a net benefit from voting, $c<0$. Such a direct benefit can manifest itself as the fear from the collapse of democracy or a sense of civic duty.

While appealing, many rational choice theorists were unwilling to accept a resolution based on "irrational" voters who value voting as a consumption good. Ledyard $(1981,1984)$ was the first to construct a game-theoretic model in which all citizens are rational and choose between two alternatives. He demonstrated the possibility of positive turnout when the net value from winning is sufficiently greater than the cost. In a pair of very influential papers, Palfrey and Rosenthal (1983, 1985) refined Ledyard's model and addressed the issue of turnout in large electorates. Specifically, Palfrey and Rosenthal (1983) assumed that the number of citizens who prefer each alternative and the (positive) cost of voting are common knowledge. They found that substantial turnout can occur in a subset of Nash equilibria. In particular, if each group is of equal size and large, then there are exactly two symmetric mixed strategy equilibria, one with almost no voting and one with almost everyone voting.

To determine the robustness of these two polar equilibria, Palfrey and Rosenthal (1985) extended their analysis to a setting where the cost of voting is private to each citizen and varies across them. In this environment, each citizen follows a simple cutoff strategy: vote if the cost is below a threshold and abstain otherwise. Thus, individual voting becomes less likely as the cutoff decreases. Indeed, Palfrey and Rosenthal showed that as the electorate size grows without bound, the cutoff cost converges to (the lower bound of ) zero. This result led Palfrey and Rosenthal to reach the startling conclusion that "... [our result] is consistent with the Downs-Riker-Ordeshook-Tullock view that the only reason a rational 
voter would vote in large elections is if the net cost is negative ..." (p. 71), "We have come full circle and are once again beset by the paradox of not voting." (p. 64.)

This negative finding has played a significant role in subsequent research. ${ }^{1}$ In their 1994 book, Green and Shapiro wrote "Game-theoretic solutions to the turnout problem have not resurfaced since this retreat [by Palfrey and Rosenthal (1985)] was sounded almost a decade ago." (p. 58.) In the same vein, many researchers have acknowledged the setback and turned their attention to alternative models of the civic-duty story first suggested by Downs (1957). For instance, in recent work, Shachar and Nalebuff (1999) consider a followthe-leader-model in which each group is composed of one leader and the followers. It is only the leader who acts strategically and expends effort to increase turnout by followers. ${ }^{2}$ Coate and Conlin (2004) provide a group-utilitarian model of turnout in which each citizen chooses a cutoff cost that maximizes the group's aggregate utility. ${ }^{3}$

In this paper, rather than introducing an alternative explanation, we take the analysis of Palfrey and Rosenthal (1985) one step further and fully characterize voter turnout in large electorates. We show that while, as in Palfrey and Rosenthal (1985), the likelihood of individual voting becomes negligible, expected voter turnout can nevertheless be significant. In particular, we show that as the lower bound of the voting cost distribution approaches zero, expected voter turnout grows without bound.

Our model is a slight variation of Palfrey and Rosenthal (1985). We assume the electorate is composed of a fixed number of ex ante identical citizens who choose between two alternatives. Each citizen is equally likely to favor either alternative, and the private cost of voting follows a continuous distribution with a positive lower bound, $\underline{c}>0$. Consistent with Palfrey and Rosenthal (1985), we find a unique symmetric equilibrium in which each citizen follows a cutoff rule. Moreover, as the electorate size goes to infinity, this cutoff converges to $\underline{c}$, implying an infinitesimal probability of voting for each citizen. However, rather than interpreting this limit result as an indication of infinitesimal expected turnout, we explicitly characterize limiting voter behavior. We show that the limit turnout follows a Poisson distribution with a mean approximately equal to $1 /\left(2 \pi \underline{c}^{2}\right) .{ }^{4}$ Hence, as $\underline{c}$ gets smaller, the expected turnout increases without bound. ${ }^{5}$

Our finding has two main implications. First, a significant number of rational citizens can turn out to vote in large elections, even when the cost of voting is strictly positive. Thus, there does not seem to be any a priori conflict between rationality and voting. Second, since the equilibrium turnout follows a Poisson distribution, the rational voter model has the potential to account for variations in actual turnout levels. However, whether this simple model of voting is consistent with the data is an empirical question. Hansen et al. (1987)

\footnotetext{
${ }^{1}$ For an overview of the literature, see Aldrich (1993, 1997), Green and Shapiro (1994, ch. 4), and Matsusaka and Palda (1993).

${ }^{2}$ Morton (1987) and Uhlaner (1989) are the first ones who postulated the role of group leaders on turnout.

${ }^{3}$ Coate and Conlin base their theory on an earlier work by Feddersen and Sandroni (2002).

${ }^{4}$ This finding is reminiscent of Myerson (1998, 2000). The important difference, however, is that the mean of the Poisson distribution in our model is endogenous and forms the heart of our analysis.

${ }^{5}$ The observation that negligible individual voting can lead to significant aggregate voting is analagous to equilibrium production in a standard Cournot oligopoly model. As the number of firms becomes large, equilibrium production by each firm converges to zero, while aggregate production is significantly positive.
} 
provided some early evidence in favor of the model, but empirical studies have often faced the difficulty of testing the small probability of being pivotal. As Coate and Conlin (2004) put it: "While there are good reasons to be skeptical about the pivotal voter model's ability to explain turnout, it represents in many respects the simplest way of thinking about voter behavior. Thus, it should be rejected only if it can be shown to be outperformed by some coherent alternative. This has yet to be demonstrated ... Given its [empirical] complexity, this will be a challenging task." (p.1496.)

The rest of the paper is organized as follows. Section 2 sets out the model. Section 3 characterizes the equilibrium for a finite electorate. Section 4 extends the characterization to a large electorate and presents the main finding of the paper. Section 5 provides a useful approximation to the expected limit turnout, followed by concluding remarks in Section 6 . Proofs that do not appear in the text are relegated to an appendix.

\section{The Model}

There are $n \geq 2$ risk-neutral agents who may cast a vote in an election between two alternatives, $A$ and $B$. Each agent $i$ privately knows his type which is two-dimensional, consisting of his political preference $t_{i} \in\{A, B\}$ and his cost of voting $c_{i} \in[\underline{c}, \bar{c}]$. If alternative $t$ wins the election, then a type $t$ agent receives a payoff normalized to 1 and a type $t^{\prime} \neq t$ agent receives a payoff normalized to 0 . (Hence, $c_{i}$ is the relative cost of voting; i.e., the absolute cost to net-benefit ratio.) Agents are ex ante symmetric. In particular, (i) political preferences are independently distributed with $\operatorname{Pr}\left\{t_{i}=A\right\}=1 / 2$ for $i=1, \ldots n$, and (ii) voting costs are also independently distributed with c.d.f. $G(c)$. In order to rule out trivial equilibria in which no agents vote, it is assumed that $\underline{c} \in(0,1 / 2)$. Agents simultaneously choose whether to vote for their preferred alternative or to abstain. (Note that abstaining weakly dominates voting for one's less preferred alternative.) The election is decided by majority rule and ties are broken by a fair coin toss.

\section{Equilibrium in Finite Electorates}

It is straightforward to verify that in a type-symmetric Bayesian Nash Equilibrium (BNE), agents follow a cutoff strategy in which a type $t$ player votes if and only if his cost is less than some critical level, $c_{t}^{*}$. In order to characterize such a BNE, define the ex ante probability that an agent votes for alternative $A$ by $\alpha_{A} \equiv(1 / 2) G\left(c_{A}^{*}\right)$ and the ex ante probability that he votes for $B$ by $\alpha_{B} \equiv(1 / 2) G\left(c_{B}^{*}\right)$. Hence, the ex ante probability that an agent abstains is $\left(1-\alpha_{A}-\alpha_{B}\right)$. Now, recall that the number of ways $k_{A}$ agents can vote for $A, k_{B}$ can vote for $B$, and $n-1-k_{A}-k_{B}$ can abstain is given by the trinomial coefficient

$$
\left(\begin{array}{c}
n-1 \\
k_{A}, k_{B}, n-1-k_{A}-k_{B}
\end{array}\right) \equiv \frac{(n-1) !}{k_{A} ! k_{B} !\left(n-1-k_{A}-k_{B}\right) !} .
$$


Given this, it can be shown that the net expected utility to a type $t$ agent from voting may be written

$$
\frac{1}{2} \widehat{P}\left(\alpha_{t}, \alpha_{t^{\prime}}, n\right)-c
$$

where

$$
\begin{aligned}
\widehat{P}\left(\alpha_{t}, \alpha_{t^{\prime}}, n\right) & \equiv \sum_{k=0}^{\left\lfloor\frac{n-1}{2}\right\rfloor}\left(\begin{array}{c}
n-1 \\
k, k, n-1-2 k
\end{array}\right) \alpha_{t}^{k} \alpha_{t^{\prime}}^{k}\left(1-\alpha_{t}-\alpha_{t^{\prime}}\right)^{n-1-2 k} \\
& +\sum_{k=0}^{\left\lfloor\frac{n-2}{2}\right\rfloor}\left(\begin{array}{c}
n-1 \\
k, k+1, n-2-2 k
\end{array}\right) \alpha_{t}^{k} \alpha_{t^{\prime}}^{k+1}\left(1-\alpha_{t}-\alpha_{t^{\prime}}\right)^{n-2-2 k},
\end{aligned}
$$

for $t=A, B, t \neq t^{\prime}$, and $\lfloor\cdot\rfloor$ is the usual operator that rounds a number to the lower integer when necessary.

To understand expression (1), observe that $\widehat{P}\left(\alpha_{t}, \alpha_{t^{\prime}}, n\right)$ is the probability that the (type $t$ ) agent in question castes a decisive vote; i.e., that his vote is pivotal in determining the outcome. Specifically, his vote may be pivotal for one of two reasons corresponding to the two summations in (2). First, if $k$ of the other agents vote for $t, k$ vote for $t^{\prime}$, and $n-2 k$ abstain, then the agent in question will break a tie by voting. The first summation in (2) is, therefore, the probability that the agent breaks a tie that would otherwise occur. Second, if $k$ agents vote for alternative $t, k+1$ vote for $t^{\prime}$, and $n-1-2 k$ abstain, then the agent in question will create a tie by voting. The second summation in (2) is, therefore, the probability that the agent in question creates a tie when alternative $t^{\prime}$ would otherwise have won. When his vote breaks a tie, the probability that alternative $t$ is implemented rises from $1 / 2$ to 1 , and when his vote creates a tie, the probability that $t$ is implemented rises from 0 to $1 / 2$. This accounts for the factor $1 / 2$ in (1). Of course, when an agent votes, his net expected benefit must also account for his voting cost $c$.

For the next step in characterizing the equilibrium, note that an agent whose cost of voting coincides with the cutoff is indifferent between voting and abstaining:

$$
\begin{aligned}
& \frac{1}{2} \widehat{P}\left(\alpha_{A}, \alpha_{B}, n\right)-c_{A}^{*}=0, \\
& \frac{1}{2} \widehat{P}\left(\alpha_{B}, \alpha_{A}, n\right)-c_{B}^{*}=0 .
\end{aligned}
$$

Since $\alpha_{t}=(1 / 2) G\left(c_{t}^{*}\right)$, we can rewrite these equations in the form

$$
\begin{aligned}
& G\left(\frac{1}{2} \widehat{P}\left(\alpha_{A}, \alpha_{B}, n\right)\right)-2 \alpha_{A}=0, \\
& G\left(\frac{1}{2} \widehat{P}\left(\alpha_{B}, \alpha_{A}, n\right)\right)-2 \alpha_{B}=0 .
\end{aligned}
$$

Finding an equilibrium, therefore, amounts to finding a pair $\left(\alpha_{A}, \alpha_{B}\right) \in[0,1 / 2] \times[0,1 / 2]$ that solves these two equations. 
Lemma 1 (Equal Cutoffs) In equilibrium, $\alpha_{A}=\alpha_{B}$.

This says that both types of agent use the same cutoff; i.e., that any type-symmetric $\operatorname{BNE}\left(c_{A}^{*}, c_{B}^{*}\right)$ must be strongly symmetric in the sense that $c_{A}^{*}=c_{B}^{*}$. Given this, the two equations implicitly defining an equilibrium collapse to the single condition

$$
G\left(\frac{1}{2} P(\alpha, n)\right)-2 \alpha=0
$$

where for notational convenience, we define the probability that a given voter is pivotal when each of the other $n-1$ agents votes for either alternative with probability $\alpha$ by

$$
\begin{aligned}
P(\alpha, n) & \equiv \widehat{P}(\alpha, \alpha, n)=\sum_{k=0}^{\left\lfloor\frac{n-1}{2}\right\rfloor}\left(\begin{array}{c}
n-1 \\
k, k, n-1-2 k
\end{array}\right) \alpha^{2 k}(1-2 \alpha)^{n-1-2 k} \\
& +\sum_{k=0}^{\left\lfloor\frac{n-2}{2}\right\rfloor}\left(\begin{array}{c}
n-1 \\
k, k+1, n-1-2 k
\end{array}\right) \alpha^{2 k+1}(1-2 \alpha)^{n-2-2 k} .
\end{aligned}
$$

Lemma 2 (The Pivotal Probability) The function $P(\alpha, n)$ satisfies the following properties:

(i) $P(0, n)=1$;

(ii) $P(\alpha, n)$ is strictly decreasing in both $\alpha \in(0,1 / 2]$ and $n$;

(iii) $\lim _{n \rightarrow \infty} P(\alpha, n)=0$ for $\alpha>0$.

This result is very intuitive. First, the probability that an agent's vote is pivotal when no-one else votes is, clearly, one. Second, it is only sensible that the probability of being pivotal is smaller when other agents vote with higher probability or when there are more agents in the electorate. Finally, as the number of agents in the electorate tends to infinity, the probability that a single agent's vote changes the outcome of the election approaches zero. With these facts in hand, we can prove the following important result.

\section{Proposition 1 (The Equilibrium Probability of Voting)}

(i) There exists a unique ex ante equilibrium probability of voting for either alternative; i.e., a unique $\alpha^{*}(n) \in[0,1 / 2]$ that solves (5).

(ii) The ex ante equilibrium probability of voting for either alternative, $\alpha^{*}(n)$, is strictly decreasing in $n$.

(iii) As the number of potential voters, $n$, tends to infinity, $\alpha^{*}(n)$ converges to zero.

Proof of Proposition 1. 
(i) Fix an $n$ and define $\Psi(\alpha) \equiv G((1 / 2) P(\alpha, n))-2 \alpha$. Since, by Lemma $2, P(\alpha, n)$ is strictly decreasing in $\alpha \in(0,1 / 2]$, so is $\Psi(\alpha)$. This means that there is at most one solution to $\Psi(\alpha)=0$. Moreover, since $P(0, n)=1$, it follows that $\Psi(0)=G(1 / 2)>0$ and $\Psi(1 / 2)=G((1 / 2) P((1 / 2), n))-1<0$. Hence, there is a unique solution $\alpha^{*}(n) \in$ $[0,1 / 2]$ that solves $(5)$.

(ii) To show that $\alpha^{*}(n)$ is strictly decreasing, it suffices to show that for a fixed $\alpha \in(0,1 / 2]$, $P(\alpha, n)$ is strictly decreasing in $n$. But this follows from Lemma 2.

(iii) Note that since $\alpha^{*}(n)$ is strictly decreasing and bounded below by zero, it must converge to some $\alpha_{\ell} \geq 0$. Suppose $\alpha_{\ell}>0$. From (5) and by continuity, this implies that $\lim _{n \rightarrow \infty} G\left((1 / 2) P\left(\alpha_{\ell}, n\right)\right)=2 \alpha_{\ell} \neq 0$. However, Lemma 2 implies that for $\alpha_{\ell}>0$, $\lim _{n \rightarrow \infty} G\left((1 / 2) P\left(\alpha_{\ell}, n\right)\right)=0$, yielding a contradiction.

In order to interpret Proposition 1, observe that the equilibrium cost cutoff is $c^{*}(n) \equiv$ $G^{-1}\left(2 \alpha^{*}(n)\right)$ (see Figure 1). It is then possible to restate Proposition 1 in the following more intuitive form.

\section{Corollary 1 (The Equilibrium Cost Cutoff)}

(i) There exists a unique equilibrium cutoff $c^{*}(n) \in[\underline{c}, 1 / 2]$ such that an agent with cost $c$ votes if and only if $c \leq c^{*}(n)$.

(ii) The equilibrium cutoff, $c^{*}(n)$, is decreasing in $n$.

(iii) As the number of potential voters, $n$, tends to infinity, $c^{*}(n)$ converges to $\underline{c}$.

Proof of Corollary 1. Given that $c^{*}(n) \equiv G^{-1}\left(2 \alpha^{*}(n)\right)$ and $G^{-1}(\bullet)$ is strictly increasing, the results follow directly from Proposition 1.

Existence and uniqueness of the equilibrium follow primarily from the fact that the probability of being pivotal is smaller when agents vote with higher frequency. Hence, $P((1 / 2) G(c), n)$ is decreasing in $c$ and there, thus, exists a unique number $c^{*}(n)$ satisfying $(1 / 2) P\left((1 / 2) G\left(c^{*}(n)\right), n\right)=c^{*}(n)$. The equilibrium cost cutoff, $c^{*}(n)$, is decreasing in $n$ because the probability of being pivotal falls when the number of agents increases, leading to a decrease in the net expected benefit from voting. (This is illustrated in Figure 1 as a downward shift in $(1 / 2) P(\cdot)$ as the number of agents rises from $n$ to $n^{\prime}$.) Since $c^{*}(n)$ is the level of cost that leaves an agent just indifferent between voting and abstaining, it must fall when $n$ increases (hence $c^{*}\left(n^{\prime}\right)<c^{*}(n)$ in Figure 1).

Finally, to see that $c^{*}(n)$ converges to $\underline{c}$, note that it cannot converge to any $c>\underline{c}$ because the net expected benefit from voting, $(1 / 2) P((1 / 2) G(c), n)$, converges to zero, implying that even an agent with voting $\operatorname{cost} \underline{c}$ would strictly prefer to abstain. Hence, Corollary 1 replicates the celebrated result of Palfrey and Rosenthal (1985) that the equilibrium cutoff cost approaches the lower bound of the support of voting costs as the number of agents in the electorate tends to infinity. ${ }^{6}$ As we noted in the Introduction, however, this does not

\footnotetext{
${ }^{6}$ As noted in the Introduction, Palfrey and Rosenthal (1985) actually assumed $\underline{c} \leq 0$ and they showed that $c^{*}(n)$ converges to zero; i.e., the fraction of agents with positive costs who vote goes to zero.
} 


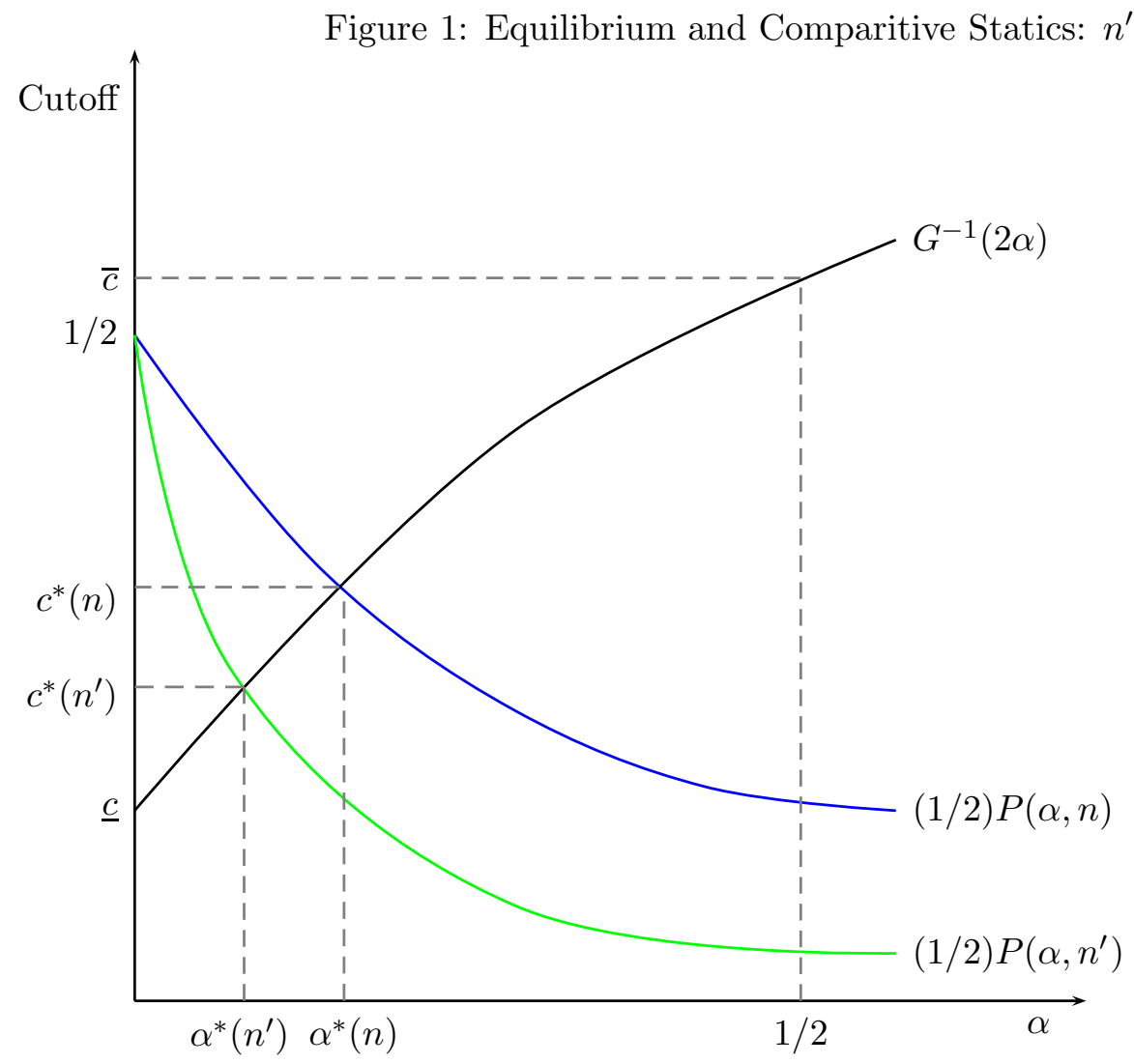

imply that no agents vote in the limit. Indeed, the number of agents that vote in the limit may actually be very large as we demonstrate in the next section.

\section{Turnout in Large Elections}

We begin this section by showing that the fact that $c^{*}(n)$ converges to $\underline{c}$ does not imply that no agent votes in the limit.

Proposition 2 In equilibrium, $\lim _{n \rightarrow \infty} \operatorname{Pr}\{$ all agents abstain $\mid n\} \neq 1$.

Proof of Proposition 2. Since the ex ante probability that each citizen votes for one of the alternatives is $\alpha^{*}(n), \operatorname{Pr}\{$ all agents abstain $\mid n\}=\left[1-2 \alpha^{*}(n)\right]^{n}$. Suppose, by way of contradiction, that

$$
\lim _{n \rightarrow \infty}\left[1-2 \alpha^{*}(n)\right]^{n}=1
$$


From part (iii) of Proposition 1 we know

$$
\lim _{n \rightarrow \infty}\left[1-\alpha^{*}(n)\right]^{-1}=1 .
$$

Multiplying the two limits then gives

$$
\lim _{n \rightarrow \infty}\left[1-2 \alpha^{*}(n)\right]^{n-1}=1 .
$$

It is easy to verify that

$$
\left[1-2 \alpha^{*}(n)\right]^{n-1} \leq P\left(\alpha^{*}(n), n\right) \leq 1,
$$

and hence

$$
\lim _{n \rightarrow \infty} P\left(\alpha^{*}(n), n\right)=1,
$$

implying that

$$
\lim _{n \rightarrow \infty} G\left(\frac{1}{2} P\left(\alpha^{*}(n), n\right)\right)=G\left(\frac{1}{2}\right)>0 .
$$

However, since $\alpha^{*}(n)$ satisfies

$$
G\left(\frac{1}{2} P\left(\alpha^{*}(n), n\right)\right)-2 \alpha^{*}(n)=0,
$$

and

$$
\lim _{n \rightarrow \infty} \alpha^{*}(n)=0,
$$

by part (iii) of Proposition 1 , we have

$$
\lim _{n \rightarrow \infty} G\left(\frac{1}{2} P\left(\alpha^{*}(n), n\right)\right)=0,
$$

yielding a contradiction.

This result implies that there is positive expected voter turnout in the limit as $n$ tends to infinity, even though the probability that a given agent votes, $2 \alpha^{*}(n)$ converges to zero. The intuition is simply that it cannot be an equilibrium for all agents to abstain, since an agent who deviated would surely be pivotal and garner a payoff of $1 / 2-c$. Since $\underline{c}<1 / 2$, there is a positive probability that any given agent will find such a deviation profitable. Indeed, it follows directly from (5) and part (iii) of Corollary 1 that

$$
\lim _{n \rightarrow \infty} P\left(\alpha^{*}(n), n\right)=2 \underline{c} .
$$

In other words, the equilibrium probability that a given voter is pivotal converges to $2 \underline{c}<1$, which also indicates that expected voter turnout must be positive. 
In order to determine expected voter turnout in the limit, consider the situation facing a representative type $t$ agent and suppose that the other $n-1$ agents vote if and only if their costs are less than the equilibrium cutoff $c^{*}(n)$. let $X_{A}$ and $X_{B}$ be the number of votes for alternatives $\mathrm{A}$ and $\mathrm{B}$, respectively. Furthermore, let $X_{0}=n-X_{A}-X_{B}$ be the number of abstentions. Using this notation, the representative agent's vote will be pivotal if and only if $X_{t^{\prime}}=X_{t}$ (he breaks a tie) or $X_{t^{\prime}}=X_{t}+1$ (he creates a tie). Hence, the probability that his vote is pivotal can be written

$$
P\left(\alpha^{*}(n), n\right)=\operatorname{Pr}\left\{X_{t^{\prime}}=X_{t}\right\}+\operatorname{Pr}\left\{X_{t^{\prime}}=X_{t}+1\right\} .
$$

Next, observe that $\left(X_{A}, X_{B}, X_{0}\right) \sim \operatorname{Multinomial}\left(\alpha^{*}(n), \alpha^{*}(n), 1-2 \alpha^{*}(n) \mid n-1\right)$.

We are interested in computing expected equilibrium turnout as $n$ tends to infinity. That is, we wish to compute

$$
\lim _{n \rightarrow \infty} 2 \alpha^{*}(n) n=\lim _{n \rightarrow \infty} E\left[X_{A}+X_{B} \mid n\right] .
$$

For notational convenience we denote this limit by $m$. In order to determine it, we next derive the asymptotic distributions of $X_{A}$ and $X_{B}$.

Lemma 3 The limiting marginal distributions of $X_{A}$ and $X_{B}$ are independent Poisson distributions with the same mean, $m / 2$. Hence, the limiting distribution of $X_{A}+X_{B}$ is Poisson with mean $m$.

Let $f(k \mid \mu)$ be the p.d.f. for a Poisson distribution with mean $\mu$. Recall that $f(k \mid \mu)=$ $\frac{\mu^{k} e^{-\mu}}{k !}$ for $k=0,1, \ldots$ Combining $(7)$ and Lemma 3 , it follows that

$$
\begin{aligned}
\lim _{n \rightarrow \infty} P\left(\alpha^{*}(n), n\right) & =\lim _{n \rightarrow \infty}\left[\operatorname{Pr}\left\{X_{t^{\prime}}=X_{t}\right\}+\operatorname{Pr}\left\{X_{t^{\prime}}=X_{t}+1\right\}\right] \\
& =\sum_{k=0}^{\infty} f^{2}\left(k \mid \frac{m}{2}\right)+\sum_{k=0}^{\infty} f\left(k \mid \frac{m}{2}\right) f\left(k+1 \mid \frac{m}{2}\right) \equiv H(m) .
\end{aligned}
$$

Finally, combining this with (6) shows that limiting expected equilibrium voter turnout must satisfy $H(m)=2 \underline{c}$.

\section{Proposition 3 (Turnout In Large Elections)}

(i) For $\underline{c} \in(0,1 / 2)$, limiting expected voter turnout is the unique number $m^{*} \in(0, \infty)$ satisfying $H\left(m^{*}\right)=2 \underline{c}$.

(ii) Expected turnout, $m^{*}$, is strictly decreasing in $\underline{c}$.

(iii) For any $M<\infty$, there exists $\widehat{c}(M)>0$ such that $\underline{c}<\widehat{c}(M)$ implies $m^{*}>M$.

This result is the central finding of the paper. It indicates that limiting expected voter turnout is not only positive, but tends to infinity as $\underline{c}$ approaches zero. Indeed, the probability that voter turnout exceeds $k$ is $1-F\left(k \mid m^{*}\right)$, where $F(k \mid \mu)$ is the $c . d . f$. of the Poisson distribution. Hence, even when $\underline{c}$ is not especially close to zero, there is still a positive probability of observing arbitrarily high voter turnout. 


\section{Approximating Expected Turnout}

In principle, the equation $H(m)=2 \underline{c}$ could be used to calculate limiting expected voter turnout, $m^{*}$, for various values of $\underline{c}$. Unfortunately, $H(m)$ is a very complicated function that cannot be computed directly, let alone inverted. ${ }^{7}$ It is possible, however, to use the Normal approximation to the Poisson distribution to obtain approximate values for expected voter turnout when $\underline{c}$ is relatively small.

Proposition 4 (Turnout Approximation) For small $\underline{c}>0$, Limiting expected voter turnout is approximated by the formula

$$
m^{*} \approx \frac{1}{2 \pi \underline{c}^{2}} .
$$

Proof of Proposition 4. Define $W=X_{t^{\prime}}-X_{t}$. From Lemma 3 , it follows that $E[W]=0$ and $\operatorname{Var}(W)=m$. By the Central Limit Theorem,

$$
\frac{W-0}{\sqrt{m}} \stackrel{D}{\longrightarrow} N(0,1)
$$

as $m \rightarrow \infty$. This implies

$$
\begin{aligned}
\lim _{n \rightarrow \infty} P\left(\alpha^{*}(n), n\right) & =\lim _{n \rightarrow \infty}[\operatorname{Pr}\{W=0\}+\operatorname{Pr}\{W=1\}] \\
& \approx \lim _{n \rightarrow \infty}[\operatorname{Pr}\{-\varepsilon \leq W \leq \varepsilon\}+\operatorname{Pr}\{1-\varepsilon \leq W \leq 1+\varepsilon\}] \text { for some } \varepsilon \in(0,1) \\
& =\operatorname{Pr}\left\{\frac{-\varepsilon}{\sqrt{m}} \leq \frac{W}{\sqrt{m}} \leq \frac{\varepsilon}{\sqrt{m}}\right\}+\operatorname{Pr}\left\{\frac{1-\varepsilon}{\sqrt{m}} \leq \frac{W}{\sqrt{m}} \leq \frac{1+\varepsilon}{\sqrt{m}}\right\} \\
& =\int_{\frac{-\varepsilon}{\sqrt{m}}}^{\frac{\varepsilon}{\sqrt{m}}} \frac{1}{\sqrt{2 \pi}} e^{-\frac{z^{2}}{2}} \mathrm{~d} z+\int_{\frac{1-\varepsilon}{\sqrt{m}}}^{\frac{1+\varepsilon}{\sqrt{m}}} \frac{1}{\sqrt{2 \pi}} e^{-\frac{z^{2}}{2}} \mathrm{~d} z \equiv \widetilde{H}(m) .
\end{aligned}
$$

Note that for $z \approx 0$, a first-order Taylor expansion reveals $e^{-z^{2} / 2} \approx 1$. Since for large values of $m, z$ is small, this observation implies

$$
\widetilde{H}(m) \approx \frac{1}{\sqrt{2 \pi}}\left[\frac{2 \varepsilon}{\sqrt{m}}+\frac{2 \varepsilon}{\sqrt{m}}\right] .
$$

Setting $\varepsilon=1 / 2$ and inverting the condition $\widetilde{H}(m)=2 \underline{c}$ yields the claim.

This result says that when the lowest possible cost realization is relatively small, expected turnout is proportional to one over its square. For instance, if $\underline{c}=0.01$, then $m^{*} \approx 1591$ and if $\underline{c}=0.0001$, then $m^{*} \approx 15.9 \times 10^{6}$. Indeed, in accordance with part (iii) of Proposition 3, approximate expected voter turnout becomes unboundedly large as $\underline{c}$ approaches zero.

\footnotetext{
${ }^{7}$ Obviously, one can approximate $H(m)$ arbitrarily closely by computing ever larger summations. Doing so is computationally demanding, but does confirm the validity of the much simpler method of approximation offered below.
} 


\section{Conclusion}

In this paper, our objective was to provide a more complete characterization of voter turnout within the standard pivotal-voter model. Specifically, we took the analysis of individual voting one step further and explicitly characterized aggregate turnout. Our main finding is that while, consistent with previous studies, the likelihood of individual voting becomes trivial in a large election, the expected turnout can nevertheless be arbitrarily large. This is so despite a positive cost of voting for all citizens. The intuition behind our finding is that the equilibrium probability of voting converges to zero much slower than the growth in electorate size. Hence, there does not seem to be a priori inconsistency between rational choice theory and voting.

As in the extant literature, an important drawback of our analysis is, however, to associate a large electorate with an infinite number of citizens. Although investigating this extreme case is analytically tractable and yields valuable insights, it is also responsible for two unsettling observations. First, the limit turnout, $m^{*}$, depends only on the lowest cost of voting and not on the cost distribution itself. Second, the percentage of expected turnout given by $\frac{2 n \alpha^{*}(n)}{n}=2 \alpha^{*}(n)$ converges to zero. Thus, it is important that future work on this topic consider the case of large but finite electorates to establish a more meaningful relationship between equilibrium voter turnout and the parameters of the model.

As noted in the Introduction, whether our addendum to the standard theory can account for actual voter turnout is ultimately an empirical question. However, even if the answer to this question turns out to be negative, our analysis indicates that rational voters could still play a significant role in determining the outcome of an election. That is, it is possible that, along with the "irrational" voters, a large number of rational citizens can turn out to vote and ultimately can sway the outcome of an election. A formal analysis in this vein also seems like a promising area for future work. 


\section{Appendix}

Proof of Lemma 1: Note that $\widehat{P}\left(\alpha_{t}, \alpha_{t^{\prime}}\right)=\Lambda_{0}+\alpha_{t^{\prime}} \Lambda_{1}$, where $\left.\Lambda_{0} \equiv \sum_{k=0}^{\left\lfloor\frac{n-1}{2}\right\rfloor} \begin{array}{c}n-1 \\ k, k, n-1-2 k\end{array}\right) \alpha_{t}^{k} \alpha_{t^{\prime}}^{k}(1-$ $\left.\alpha_{t}-\alpha_{t^{\prime}}\right)^{n-1-2 k}$, and $\Lambda_{1} \equiv \sum_{k=0}^{\left\lfloor\frac{n-2}{2}\right\rfloor}\left(\begin{array}{c}n-1 \\ k, k+1, n-2-2 k\end{array}\right) \alpha_{t}^{k} \alpha_{t^{\prime}}^{k}\left(1-\alpha_{t}-\alpha_{t^{\prime}}\right)^{n-2-2 k}$. Hence, (3) and (4) are equivalent to

$$
\begin{aligned}
& G\left(\frac{1}{2}\left(\Lambda_{0}+\alpha_{B} \Lambda_{1}\right)\right)-2 \alpha_{A}=0, \\
& G\left(\frac{1}{2}\left(\Lambda_{0}+\alpha_{A} \Lambda_{1}\right)\right)-2 \alpha_{B}=0 .
\end{aligned}
$$

Suppose, w.o.l.g. that $\alpha_{B}>\alpha_{A}$. Given that $\Lambda_{1}>0$, this implies $G\left(\frac{1}{2}\left(\Lambda_{0}+\alpha_{B} \Lambda_{1}\right)\right)>$ $G\left(\frac{1}{2}\left(\Lambda_{0}+\alpha_{A} \Lambda_{1}\right)\right)$. Using the two equations, this further implies that $\alpha_{A} \geq \alpha_{B}$, yielding a contradiction. Hence, $\alpha_{A}=\alpha_{B}$.

Proof of Lemma 2: Each part is proven in turn.

(i) Note that when evaluated at $\alpha=0$, the first and second summations in $P(\alpha, n)$ reduce to 1 and 0 , respectively. Thus, $P(0, n)=1$.

(ii) To show that $P(\alpha, n)$ is strictly decreasing in $\alpha$, we simply differentiate $P(\alpha, n)$ with respect to $\alpha$

$$
\begin{aligned}
\frac{\partial}{\partial \alpha} P(\alpha, n) & =\left[\sum_{k=1}^{\left\lfloor\frac{n-1}{2}\right\rfloor} \frac{(n-1) !}{(k !)^{2}(n-1-2 k) !} 2 k \alpha^{2 k-1}(1-2 \alpha)^{n-1-2 k}\right. \\
& -\left[\sum_{k=0}^{\left\lfloor\frac{n-1}{2}\right\rfloor} \frac{(n-1) !}{(k !)^{2}(n-1-2 k) !} \alpha^{2 k} 2(n-1-2 k)(1-2 \alpha)^{n-2-2 k}\right] \\
+ & {\left[\sum_{k=0}^{\left\lfloor\frac{n-2}{2}\right\rfloor} \frac{(n-1) !}{k !(k+1) !(n-2-2 k) !}(2 k+1) \alpha^{2 k}(1-2 \alpha)^{n-2-2 k}\right.} \\
& \left.-\sum_{k=0}^{\left\lfloor\frac{n-2}{2}\right\rfloor} \frac{(n-1) !}{k !(k+1) !(n-2-2 k) !} \alpha^{2 k} 2(n-2-2 k)(1-2 \alpha)^{n-3-2 k}\right] .
\end{aligned}
$$

Next, we combine the first summation with the last one and the second summation with 
the third one,

$$
\begin{aligned}
& \frac{\partial}{\partial \alpha} P(\alpha, n) \leq(n-1) !\left[\sum_{k=1}^{\left\lfloor\frac{n-1}{2}\right\rfloor} \frac{2 k}{(k !)^{2}(n-1-2 k) !} \alpha^{2 k-1}(1-2 \alpha)^{n-1-2 k}\right. \\
& \left.\quad-\sum_{k=0}^{\left\lfloor\frac{n-2}{2}\right\rfloor} \frac{2(n-2-2 k)}{k !(k+1) !(n-2-2 k) !} \alpha^{2 k+1}(1-2 \alpha)^{n-3-2 k}\right] \\
& \quad+(n-1) ! \sum_{k=0}^{\left\lfloor\frac{n-2}{2}\right\rfloor}\left(\frac{2 k+1}{k !(k+1) !(n-2-2 k) !}-\frac{2(n-1-2 k)}{(k !)^{2}(n-1-2 k) !}\right) \alpha^{2 k}(1-2 \alpha)^{n-2-2 k} \\
& \quad=(n-1) !\left[\sum_{k=0}^{\left\lfloor\frac{n-1}{2}\right\rfloor-1} \frac{2(k+1)}{((k+1) !)^{2}(n-1-2(k+1)) !} \alpha^{2 k+1}(1-2 \alpha)^{n-3-2 k}\right. \\
& \left.\quad-\sum_{k=0}^{\left\lfloor\frac{n-2}{2}\right\rfloor} \frac{2(n-2-2 k)}{k !(k+1) !(n-2-2 k) !} \alpha^{2 k+1}(1-2 \alpha)^{n-3-2 k}\right] \\
& \quad+(n-1) ! \sum_{k=0}^{\left\lfloor\frac{n-2}{2}\right\rfloor}\left(\frac{2 k+1}{k !(k+1) !(n-2-2 k) !}-\frac{2}{(k !))^{2}(n-2-2 k) !}\right) \alpha^{2 k}(1-2 \alpha)^{n-2-2 k} \\
& +(n-1) ! \sum_{k=0}^{\left\lfloor\frac{n-2}{2}\right\rfloor}\left(\frac{2 k}{k !(k+1) !(n-2-2 k) !}-\frac{2(k+1)}{(k !))^{2}(n-2-2 k) !}\right) \alpha^{2 k}(1-2 \alpha)^{n-2-2 k} . \\
& \leq(n-1) ! \sum_{k=0}\left(\frac{n-1}{k !(k+1) !(n-2-2 k) !}-\frac{2(n+1) !)^{2}(n-1-2(k+1)) !}{((k+1}\right) \alpha^{2 k+1}(1-2 \alpha)^{n-3-2 k}
\end{aligned}
$$

Because

$$
\frac{2(k+1)}{((k+1) !)^{2}(n-1-2(k+1)) !}-\frac{2(n-2-2 k)}{k !(k+1) !(n-2-2 k) !}=0
$$

and

$$
\frac{2 k+1}{k !(k+1) !(n-2-2 k) !}-\frac{2}{(k !)^{2}(n-2-2 k) !}=-\frac{1}{k !(k+1) !(n-2-2 k) !},
$$

it follows that

$$
\frac{\partial}{\partial \alpha} P(\alpha, n) \leq-(n-1) ! \sum_{k=0}^{\left\lfloor\frac{n-2}{2}\right\rfloor} \frac{1}{k !(k+1) !(n-2-2 k) !} \alpha^{2 k}(1-2 \alpha)^{n-2-2 k}<0 .
$$


Next, to show that for $\alpha \in\left(0, \frac{1}{2}\right], P(\alpha, n)$ is strictly decreasing in $n$, note that

$$
\begin{aligned}
P(\alpha, n) & -P(\alpha, n+1)=\sum_{k=0}^{\left\lfloor\frac{n-1}{2}\right\rfloor} \frac{(n-1) !}{(k !)^{2}(n-1-2 k) !} \alpha^{2 k}(1-2 \alpha)^{n-1-2 k} \\
& +\sum_{k=0}^{\left\lfloor\frac{n-2}{2}\right\rfloor} \frac{(n-1) !}{k !(k+1) !(n-2-2 k) !} \alpha^{2 k+1}(1-2 \alpha)^{n-2-2 k}-\sum_{k=0}^{\left\lfloor\frac{n}{2}\right\rfloor} \frac{n !}{(k !)^{2}(n-2 k) !} \alpha^{2 k}(1-2 \alpha)^{n-2 k} \\
& -\sum_{k=0}^{\left\lfloor\frac{n-1}{2}\right\rfloor} \frac{n !}{k !(k+1) !(n-1-2 k) !} \alpha^{2 k+1}(1-2 \alpha)^{n-1-2 k} .
\end{aligned}
$$

Before signing this expression, we suppose that $n$ is odd, and re-write the third summation:

$$
\begin{aligned}
& \sum_{k=0}^{\left\lfloor\frac{n}{2}\right\rfloor} \frac{n !}{(k !)^{2}(n-2 k) !} \alpha^{2 k}(1-2 \alpha)^{n-2 k} \\
& \quad=\sum_{k=0}^{\frac{n-1}{2}}\left[1+\frac{2 k}{n-2 k}\right] \frac{(n-1) !}{(k !)^{2}(n-1-2 k) !} \alpha^{2 k}(1-2 \alpha)^{n-2 k} \\
& \quad=(1-2 \alpha) \sum_{k=0}^{\frac{n-1}{2}} \frac{(n-1) !}{(k !)^{2}(n-1-2 k) !} \alpha^{2 k}(1-2 \alpha)^{n-1-2 k} \\
& \quad+2 \alpha \sum_{k=1}^{\frac{n-1}{2}} \frac{(n-1) !}{(k-1) ! k !(n-2 k) !} \alpha^{2 k-1}(1-2 \alpha)^{n-2 k} \\
& \quad=(1-2 \alpha) \sum_{k=0}^{\frac{n-1}{2}} \frac{(n-1) !}{(k !)^{2}(n-1-2 k) !} \alpha^{2 k}(1-2 \alpha)^{n-1-2 k} \\
& \quad+2 \alpha \sum_{k=0}^{\frac{n-1}{2}-1} \frac{(n-1) !}{k !(k+1) !(n-2-2 k) !} \alpha^{2 k+1}(1-2 \alpha)^{n-2-2 k}
\end{aligned}
$$


Inserting this into (A-1) and cancelling terms yield

$$
\begin{aligned}
P(\alpha, n)- & P(\alpha, n+1)=2 \alpha \sum_{k=0}^{\frac{n-1}{2}} \frac{(n-1) !}{(k !)^{2}(n-1-2 k) !} \alpha^{2 k}(1-2 \alpha)^{n-1-2 k} \\
& +(1-2 \alpha) \sum_{k=0}^{\frac{n-1}{2}-1} \frac{(n-1) !}{k !(k+1) !(n-2-2 k) !} \alpha^{2 k+1}(1-2 \alpha)^{n-2-2 k} \\
& -\sum_{k=0}^{\frac{n-1}{2}} \frac{n !}{k !(k+1) !(n-1-2 k) !} \alpha^{2 k+1}(1-2 \alpha)^{n-1-2 k} \\
= & \sum_{k=0}^{\frac{n-1}{2}-1}\left(\frac{2}{n-1-2 k}+\frac{1}{k+1}\right) \frac{(n-1) !}{(k !)^{2}(n-2-2 k) !} \alpha^{2 k+1}(1-2 \alpha)^{n-1-2 k} \\
& -\sum_{k=0}^{\frac{n-1}{2}} \frac{n !}{k !(k+1) !(n-1-2 k) !} \alpha^{2 k+1}(1-2 \alpha)^{n-1-2 k}+\frac{2(n-1) !}{\left(\frac{n-1}{2} !\right)^{2}} \alpha^{n} \\
= & \sum_{k=0}^{n-1}-1 \\
+ & \frac{2(n-1) !}{\left(\frac{n-1}{2} !\right)^{2}} \alpha^{n}-\frac{(n-1) !}{\frac{n-1}{2} !\left(\frac{n-1}{2}+1\right) !} \alpha^{n}>0 .
\end{aligned}
$$

The exact arguments prove the result for $n$ even.

(iii) Fix any $\alpha \in\left(0, \frac{1}{2}\right]$. Since $P(\alpha, n)$ is decreasing in $n$ and bounded below by 0 , it converges to some $\psi(\alpha) \geq 0$ and so does its subsequence $P(\alpha, n+1)$, where, by definition

$$
\begin{aligned}
P(\alpha, n+1) & =\sum_{k=0}^{\frac{n}{2}} \frac{n !}{(k !)^{2}(n-2 k) !} \alpha^{2 k}(1-2 \alpha)^{n-2 k} \\
& +\sum_{k=0}^{\frac{n-1}{2}} \frac{n !}{k !(k+1) !(n-1-2 k) !} \alpha^{2 k+1}(1-2 \alpha)^{n-1-2 k} \\
& =(1-2 \alpha)\left[\sum_{k=0}^{\frac{n}{2}} \frac{n}{n-2 k} \frac{(n-1) !}{(k !)^{2}(n-1-2 k) !} \alpha^{2 k}(1-2 \alpha)^{n-1-2 k}\right. \\
& \left.+\sum_{k=0}^{\frac{n-1}{2}} \frac{n}{n-1-2 k} \frac{(n-1) !}{k !(k+1) !(n-2-2 k) !} \alpha^{2 k+1}(1-2 \alpha)^{n-2-2 k}\right] .
\end{aligned}
$$

Suppose $\psi(\alpha)>0$. Since, for a fixed $k, \lim _{n \rightarrow \infty} \frac{n}{n-2 k}=\lim _{n \rightarrow \infty} \frac{n}{n-1-2 k}=1$, we have $\lim _{n \rightarrow \infty} P(\alpha, n+1)=(1-2 \alpha) \lim _{n \rightarrow \infty} P(\alpha, n)$, or, equivalently $2 \alpha \psi(\alpha)=0$, yielding $\psi(\alpha)=0$, a contradiction. Hence, $\lim _{n \rightarrow \infty} P(\alpha, n)=0$. 
Proof of Lemma 3: Note that $\left(X_{A} \mid X_{B}\right) \sim \operatorname{Binomial}\left(n-1-X_{B}, \frac{\alpha^{*}(n)}{1-\alpha^{*}(n)}\right)$. Since

$$
\lim _{n \rightarrow \infty}\left[\left(n-1-X_{B}\right) \frac{\alpha^{*}(n)}{1-\alpha^{*}(n)}\right]=\frac{m}{2}
$$

it follows (see Billingsley 1995, Theorem 23.2) that

$$
\left(X_{A} \mid X_{B}\right) \stackrel{D}{\longrightarrow} \operatorname{Poisson}\left(\frac{m}{2}\right),
$$

which is independent of $X_{B}$. The same argument shows

$$
\left(X_{B} \mid X_{A}\right) \stackrel{D}{\longrightarrow} \operatorname{Poisson}\left(\frac{m}{2}\right) .
$$

Hence, the limiting distributions of $X_{A}$ and $X_{B}$ are independent Poissons. This further implies that

$$
\left(X_{A}+X_{B}\right) \stackrel{D}{\longrightarrow} \operatorname{Poisson}(m) .
$$

Proof of Proposition 3: Each part is proven in turn.

(i) We first establish that $H(m)$ is strictly decreasing in $m$. Differentiation yields

$$
\begin{aligned}
\frac{\partial}{\partial m} H(m) & =e^{-m}\left[\sum_{k=1}^{\infty} \frac{1}{2^{2 k}} \frac{2 k m^{2 k-1}}{(k !)^{2}}+2 \sum_{k=0}^{\infty} \frac{1}{2^{2(k+1)}} \frac{(2 k+1) m^{2 k}}{k !(k+1) !}\right. \\
& \left.-\sum_{k=0}^{\infty} \frac{1}{2^{2 k}} \frac{m^{2 k}}{(k !)^{2}}-2 \sum_{k=0}^{\infty} \frac{1}{2^{2(k+1)}} \frac{m^{2 k+1}}{k !(k+1) !}\right] \\
& =e^{-m}\left[\sum_{k=0}^{\infty} \frac{1}{2^{2(k+1)}} \frac{2(k+1) m^{2(k+1)-1}}{((k+1) !)^{2}}+2 \sum_{k=0}^{\infty} \frac{1}{2^{2(k+1)}} \frac{(2 k+1) m^{2 k}}{k !(k+1) !}\right. \\
& \left.-\sum_{k=0}^{\infty} \frac{1}{2^{2 k}} \frac{m^{2 k}}{(k !)^{2}}-2 \sum_{k=0}^{\infty} \frac{1}{2^{2(k+1)}} \frac{m^{2 k+1}}{k !(k+1) !}\right] .
\end{aligned}
$$

Since the first and last summations cancel out, we have

$$
\begin{gathered}
\frac{\partial}{\partial m} H(m)=e^{-m}\left[2 \sum_{k=0}^{\infty} \frac{1}{2^{2(k+1)}} \frac{(2 k+1) m^{2 k}}{k !(k+1) !}-\sum_{k=0}^{\infty} \frac{1}{2^{2 k}} \frac{m^{2 k}}{(k !)^{2}}\right] \\
=e^{-m} \sum_{k=0}^{\infty}\left[\frac{\frac{1}{2}(2 k+1)}{k+1}-1\right] \frac{1}{2^{2 k}} \frac{m^{2 k}}{(k !)^{2}}<0 .
\end{gathered}
$$

Next, it is easy to verify that $H(0)=1$ and $\lim _{m \rightarrow \infty} H(m)=0$, which imply

$$
H(0)-2 \underline{c}>0 \text { and } \lim _{m \rightarrow \infty}[H(m)-2 \underline{c}]<0 .
$$

Hence, there is a unique $m^{*} \in(0, \infty)$ that satisfies $H\left(m^{*}\right)=2 \underline{c}$. 
(ii) The fact that $\frac{\partial}{\partial m} H(m)<0$ implies that $m^{*}$ is strictly decreasing in $\underline{c}$.

(iii) Fix $M<\infty$ and define

$$
\widehat{c}(M) \equiv \frac{1}{2} H(M) .
$$

Since $M$ is finite and

$$
\lim _{m \rightarrow \infty} H(m)=0
$$

$\widehat{c}(M)>0$. Furthermore, since $m^{*}$ is strictly decreasing in $\underline{c} \in\left(0, \frac{1}{2}\right)$, the result follows. 


\section{References}

[1] Aldrich, J. "Rational Choice and Turnout," American Journal of Political Science, 1993, 37(1), 246-79.

[2] Aldrich, J. "When is It Rational to Vote?" in D.C. Mueller, Perspectives on Public Choice: A Handbook. Cambridge: Cambridge University Press, 1997, 373-90.

[3] Billingsley, P., Probability and Measure, 1995, John Wiley \& Sons.

[4] Coate, S., And M. Conlin. "A Group-Utilitarian Approach to Voter Turnout: Theory and Evidence," American Economic Review, 94(3), 1476-504.

[5] Downs, A. An Economic Theory of Democracy. 1957. New York: Harper and Row.

[6] Feddersen, T., And A. Sandroni. "A Theory of Participation in Elections," mimeo, 2002.

[7] Green, D., And I. Shapiro. Pathologies of Rational Choice Theory: A Critique of Aplications in Political Science. New Haven: Yale University Press, 1994.

[8] Hansen, S., T. Palfrey, and H. Rosenthal. "The Downsian Model of Electoral Participation: Formal Theory and Empirical Analysis of the Constituency Size Effect," Public Choice, 1987, 52(1), 15-33.

[9] Ledyard, J. "The Paradox of Voting and Candidate Competition: A General Equilibrium Analysis, " in Essays in Contemporary Fields of Economics. 1981. Eds.: G. Horwich and J. Quirk. Purdue University Press.

[10] Ledyard, J., "The Pure Theory of Large Two-Candidate Elections," Public Choice, 1984, 44(1), 7-41.

[11] Matsusaka, J., And F. Palda. "The Downsian Voter Meets the Ecological Fallacy," Public Choice, 1993, 77, 855-78.

[12] Morton, R. "A Group Majority Voting Rule of Public Good Provision," Social Choice and Welfare, 1987, 4, 117-31.

[13] Myerson, R. "Population Uncertainty and Poisson Games," International Journal of Game Theory, 1998, 27, 375-92.

[14] Myerson, R. "Large Poisson Games," Journal of Economic Theory, 2000, 94, 7-45.

[15] Palfrey, T., and H. Rosenthal. "A Strategic Calculus of Voting," Public Choice, $1983,41,7-53$.

[16] Palfrey, T., and H. Rosenthal. "Voter Participation and Strategic Uncertainty," American Political Science Review, 1985, 79, 62-78. 
[17] Riker, W., And P. Ordeshook. "A Theory of the Calculus of Voting," American Political Science Review, 1968, 62(1), 25-42.

[18] Shachar, R. And B. Nalebuff. "Follow the Leader: Theory and Evidence on Political Participation," American Economic Review, 1999, 89(3), 525-47.

[19] Uhlaner, C. "Rational Turnout: The Neglected Role of Groups," American Journal of Political Science, 1989, 33(2), 390-422. 